\title{
Does continuous glucose monitoring during pregnancy improve glycaemic and health outcomes in women with type 1 diabetes? - what the CONCEPTT trial adds
}

\author{
Diane Farrar ${ }^{1}$, Matthew D. Campbell ${ }^{2}$ \\ ${ }^{1}$ Bradford Institute for Health Research, Bradford Royal Infirmary, Bradford, UK; ${ }^{2}$ Institute of Sport, Physical Activity, and Leisure, Leeds Beckett \\ University, Leeds, UK \\ Correspondence to: Diane Farrar. Bradford Institute for Health Research, Bradford Royal Infirmary, Bradford BD9 6RJ, UK Department of Health \\ Sciences, University of York, York YO10 5DD, UK. Email: Diane.Farrar@bthft.nhs.uk. \\ Provenance: This is an invited Editorial commissioned by Section Editor Kaiping Zhang (AME College, AME Group, Hangzhou, China) and Guest \\ Section Editor Hengrui Liang (Nanshan Clinical Medicine School, Guangzhou Medical University, Guangzhou, China). \\ Comment on: Feig DS, Donovan LE, Corcoy R, et al. Continuous glucose monitoring in pregnant women with type 1 diabetes (CONCEPTT): a \\ multicentre international randomised controlled trial. Lancet 2017;390:2347-59.
}

Submitted Feb 08, 2018. Accepted for publication Feb 28, 2018.

doi: $10.21037 / \mathrm{atm} .2018 .03 .08$

View this article at: http://dx.doi.org/10.21037/atm.2018.03.08

Women with type 1 diabetes are at increased risk of adverse pregnancy outcomes including: miscarriage, congenital anomalies, pre-eclampsia, preterm birth, large for gestational age and admission to neonatal intensive care, compared to women without type 1 diabetes (1-3). The degree of risk is related to inadequate glucose control, determined by increasing glycated haemoglobin $\mathrm{A}_{1 \mathrm{c}}$ $\left(\mathrm{HbA}_{1 \mathrm{C}}\right)$ levels $(1,4)$, and/or increased placental and vascular reactivity (5). The relationship between glucose variability and adverse outcomes in pregnancy is less clear (6). $\mathrm{HbA}_{1 \mathrm{c}}$ provides an average estimate of glycaemic status over the previous three months (7). In the UK, the National Institute for Health and Care Excellence (NICE) suggest $\mathrm{HbA}_{1 \mathrm{c}}$ in pregnancy can be used to assess the degree of adverse outcome risk, but NICE do not recommend $\mathrm{HbA}_{\mathrm{lc}}$ measurement to assess blood glucose control (8). Similarly, the American Diabetes Association (ADA) suggests that $\mathrm{HbA}_{1 \mathrm{c}}$ should only be used as a secondary measure to aid glycaemic control after self-monitoring of blood glucose. Therefore, pregnant women mostly use intermittent glucose monitoring to assess their general control and immediate glucose levels and adjust insulin dose in response.

Continuous glucose monitoring (CGM) is gaining popularity, via a small device CGM provides measures of interstitial glucose and involves either the application of a skin sensor, the application of a subcutaneous sensor, which can remain in situ for several days, or the implantation of a sensor which can last up to three months. CGM devices provide real time estimates of glucose, allowing immediate adjustment of insulin dose and can be used in conjunction with continuous subcutaneous insulin infusion or multiple daily injections of insulin (9). Trials in non-pregnant populations suggest CGM compared to intermittent monitoring is associated with improved $\mathrm{HbA}_{1 \mathrm{c}}$ (10-12). There is however limited clinical- and cost-effectiveness evidence to support the use of CGM in pregnancy, a recent systematic review (13) was able to include only two trials $(14,15)$ that directly evaluated CGM with intermittent glucose monitoring. The review reported lower mean $\mathrm{HbA}_{1 \mathrm{c}}$ in the CGM group, no additional measures of glucose control were reported or measures of health service costs. There were no differences in any reported perinatal outcomes (13). The included trials were small and the evidence was assessed as being low to moderate quality.

Published in the Lancet in September 2017 Denice Feig and colleagues report the findings of CONCEPTT which included two well-designed parallel trials of CGM for women with type 1 diabetes. The trials were for women (I) planning a pregnancy or (II) already pregnant (16). CONCEPTT recruited women 18-40 years old, who were receiving intensive insulin therapy for their type 1 diabetes. The women could use either continuous subcutaneous insulin 
infusion or multiple daily injections and were randomised to receive either CGM in addition to intermittent capillary glucose monitoring or intermittent capillary glucose monitoring alone.

Outcomes included several measures of glucose control and health. The primary outcome was difference in change in $\mathrm{HbA}_{1 \mathrm{c}}$ from randomisation (women were eligible if less than 14 weeks' gestation for the pregnancy trial) to 34 weeks' gestation, and to 24 weeks or conception in the planning a pregnancy trial. To achieve the necessary power to detect a between group mean difference in $\mathrm{HbA}_{1 \mathrm{c}}$ of $0.5 \%, 215$ pregnant and 110 planning pregnancy participants were recruited. Secondary outcomes included measures of glycaemic control including: time in glucose target range, number of hypoglycaemic episodes and glycaemic variability including: standard deviation, mean amplitude of glucose excursion and rate of change and measures of maternal and infant health including: gestational hypertension and pre-eclampsia, caesarean section, large for gestational age, neonatal hypoglycaemia requiring intravenous dextrose and more than 24 hours admission to the neonatal intensive care unit.

The pregnancy trial did not demonstrate the magnitude of between group difference in change in $\mathrm{HbA}_{1 \mathrm{c}}$ anticipated, it did however show a small but statistically significant difference favouring CGM over intermittent glucose monitoring $(-0.19 \%, 95 \%$ CI: -0.34 to $-0.03 ; \mathrm{P}=0.02)$. The planning a pregnancy trial demonstrated a similar between group difference in change in $\mathrm{HbA}_{1 \mathrm{c}}$, but because of the smaller sample size (compared to the pregnancy trial) and consequent lack of power, the confidence intervals were wider and included the null value $(-0.17 \%, 95 \%$ CI: -0.43 to $0.09 ; \mathrm{P}=0.20)$. Group health outcomes in the planning a pregnancy trial was similar.

The usefulness of $\mathrm{HbA}_{1 \mathrm{c}}$ in the assessment of glycaemic control in the non-pregnant population is well documented $(10,11,17)$, but knowledge of pregnancy related haematological changes are required when interpreting $\mathrm{HbA}_{1 \mathrm{c}}$ in pregnancy and few studies have provided pregnancy specific reference ranges (18). Furthermore $\mathrm{HbA}_{1 \mathrm{c}}$ levels drop in early pregnancy in concert with increased red blood cell turn over and $\mathrm{HbA}_{1 \mathrm{c}}$ levels seem to rise in late pregnancy in most women, but may fall in some, additionally levels rise with untreated iron deficiency anaemia, a common pregnancy condition (18-20). These well recognised limitations make $\mathrm{HbA}_{1 \mathrm{c}}$ less useful in the assessment of glycaemic control in pregnancy.

In spite of the limitations of $\mathrm{HbA}_{1 \mathrm{c}}$ and small between group difference in change in $\mathrm{HbA}_{1 \mathrm{c}}$ at 34 weeks' gestation, women in the pregnancy CGM group spent more time in recommended glucose target range, and less time above target range than women using intermittent capillary glucose monitoring alone; unfortunately episodes of hypoglycaemia were not improved with CGM use. Group maternal secondary health outcomes did not differ, possibly because maternal health outcomes are more likely to be influenced by underlying physiological responses to pregnancy and less likely to be influenced by the relatively smaller differences in glucose control demonstrated in this trial (5). There were however, significant differences in some important neonatal outcomes; large for gestational age, neonatal hypoglycaemia, need for high level neonatal care $>24$ hours and infant length of hospital stay favouring CGM; all other reported neonatal outcomes rates were similar. It seems implausible that the small difference in $\mathrm{HbA}_{\mathrm{lc}}$ which is arguably clinically insignificant, resulted in the improvements in the neonatal outcome rates reported. A contributing influence however may be the quiescence of glucose levels and an avoidance of 'swings' in glucose that was shown at 34 weeks gestation in the CGM compared to the control group i.e., the CGM group demonstrated an increase in glucose control and reduction in glucose variability (reflected by more time in target, less time above target measures, standard deviation, mean amplitude of glucose excursion and rate of change). Furthermore the CGM group attended a greater number of scheduled and unscheduled visits, and, although these visits were attributed to sensor issues rather than diabetes management (pregnancy specific visits were not reported), these contacts may have contributed to the improvement in neonatal outcomes observed in the CGM group (21).

Despite the probability that the women included in these trials were more motivated than the general planning a pregnancy or pregnant diabetic population (by virtue of taking part in a trial of this nature), only $70 \%$ of women in the pregnancy and $77 \%$ in the planning a pregnancy group used CGM more than $75 \%$ of the time. This is perhaps even more surprising given that around $50 \%$ of participants in both trials used continuous subcutaneous insulin infusion pumps, an insulin delivery system that requires a high level of motivation and care and encourages greater glucose surveillance, particularly for women using pumps with inbuilt CGM systems. Clearly reduced compliance will limit the effectiveness of CGM and its impact on outcomes and accordingly compliance should be maximised. Feig and colleagues (16) report only that CGM 
users were trained and instructed to use the CGM devices daily, they do not report the requested duration of use or if any processes were put in place to monitor or increase compliance. Additionally, many more women using CGM (compared to the control group) experienced adverse events (mainly skin reactions) which may have contributed to the low compliance with CGM. It is not possible to assess the compliance of intermittent glucose monitoring because Feig and colleagues (16) only report that both CGM and control groups were advised to test capillary glucose at least seven times a day (before and one to two hours after a meal), information is absent concerning the actual frequency of intermittent monitoring conducted. This is important because the frequency of intermittent monitoring may influence $\mathrm{HbA}_{1 \mathrm{c}}$ particularly in conjunction with healthcare provider contact $(22,23)$, and as mentioned previously, scheduled and unscheduled visits with health care providers were increased in the CGM group. Evidence for the association between frequency of intermittent monitoring in pregnancy and $\mathrm{HbA}_{1 \mathrm{c}}$ is limited however (13).

Unfortunately, $20 \%$ of the $\mathrm{HbA}_{1 \mathrm{c}}$ measurements and CGM datasets were missing, these losses were within prespecified limits and the results were similar for the imputed and complete datasets, suggesting little effect; however it would be important to try to reduce these losses in future trials.

To conclude, CONCEPTT included women who were planning a pregnancy and women already pregnant, half of the participants used continuous insulin infusion, which is a high proportion that may be unrepresentative of many countries or centres and may reflect a uniquely motivated and educated group of women. Compared to women using intermittent monitoring, pregnant women randomised to use CGM demonstrated a greater reduction in $\mathrm{HbA}_{1 \mathrm{c}}$ and improved measures of glucose control and variability at 34 weeks gestation, and although maternal outcomes were similar, there were benefits in terms of some important neonatal outcomes.

Unfortunately, some information is unavailable, notably the frequency of intermittent glucose monitoring which may have influenced the primary outcome. Also at a time when health services are under increasing pressure it is important that information on the cost-effectiveness of an intervention is reported by trials. In CONCEPTT, CGM use was associated with more health care provider visits which are expensive and costs associated with CGM use (device and consumables) are considerably greater than intermittent monitoring, however, CGM was associated with health benefits for the infant that may outweigh the increased costs. Overall this trial has provided encouraging results that suggests using CGM benefits women in pregnancy both in terms of glucose control and health outcomes for their infant.

Further trials should be conducted to confirm these findings, that consider differences in insulin delivery systems (pens versus pumps for example), use new generation CGM that provide hypoglycaemia alarms and for women using continuous subcutaneous insulin infusion, an insulin suspend facility should be included. The trials should report frequency of intermittent glucose monitoring, provide interventions to increase compliance, and collect information on costs to the health services and the individual.

\section{Acknowledgements}

None.

\section{Footnote}

Conflicts of Interest: The authors have no conflicts of interest to declare.

\section{References}

1. Suhonen L, Hiilesmaa V, Teramo K. Glycaemic control during early pregnancy and fetal malformations in women with Type I diabetes mellitus. Diabetologia 2000;43:79-82 .

2. Evers IM, de Valk HW, Visser GHA. Risk of complications of pregnancy in women with type 1 diabetes: nationwide prospective study in the Netherlands. BMJ 2004;328:915.

3. Lin SF, Kuo CF, Chiou MJ, et al. Maternal and fetal outcomes of pregnant women with type 1 diabetes, a national population study. Oncotarget 2017;8:80679-87.

4. Ylinen K, Aula P, Stenman UH, et al. Risk of minor and major fetal malformations in diabetics with high haemoglobin A1c values in early pregnancy. BMJ 1984;289:345-6.

5. Colatrella A, Loguercio V, Mattei L, et al. Hypertension in diabetic pregnancy: impact and long-term outlook. Best Pract Res Clin Endocrinol Metab 2010;24:635-51.

6. Dalfrà MG, Chilelli NC, Di Cianni G, et al. Glucose Fluctuations during Gestation: An Additional Tool for Monitoring Pregnancy Complicated by Diabetes. Int J Endocrinol 2013;2013:279021.

7. Hughes RCE, Rowan J, Florkowski CM. Is There a Role 
for HbA1c in Pregnancy? Current Diabetes Reports 2016;16:5.

8. National Institute for Health and Care Excellence. Diabetes in pregnancy: management of diabetes and its complications from preconception to the postnatal period. National collaborating centre for Women's and Children's Health 2015. Available online: https://www.nice.org.uk/ guidance/ng3

9. Rodbard D. Continuous Glucose Monitoring: A Review of Recent Studies Demonstrating Improved Glycemic Outcomes. Diabetes Technology Therapeutics 2017;19:S25-37.

10. Klonoff DC. Continuous Glucose Monitoring Roadmap for 21 st century diabetes therapy. Diabetes Care 2005;28:1231-9.

11. Ahmad J, Rafat D. HbA1c and iron deficiency: A review. Diabetes Metab Syndr 2013;7:118-22.

12. Pickup JC, Freeman SC, Sutton AJ. Glycaemic control in type 1 diabetes during real time continuous glucose monitoring compared with self monitoring of blood glucose: meta-analysis of randomised controlled trials using individual patient data. BMJ 2011;343:d3805.

13. Moy FM, Ray A, Buckley BS, et al. Techniques of monitoring blood glucose during pregnancy for women with pre-existing diabetes. Cochrane Database Syst Rev 2017;6:CD009613.

14. Murphy HR, Rayman G, Lewis K, et al. Effectiveness of continuous glucose monitoring in pregnant women with diabetes: randomised clinical trial. BMJ 2008;337:a1680.

15. Secher AL, Ringholm L, Andersen HU, et al. The Effect of Real-Time Continuous Glucose Monitoring in Pregnant Women With Diabetes: A randomized controlled trial. Diabetes Care 2013;36:1877-83.

Cite this article as: Farrar D, Campbell MD. Does continuous glucose monitoring during pregnancy improve glycaemic and health outcomes in women with type 1 diabetes? - what the CONCEPTT trial adds. Ann Transl Med 2018;6(10):188. doi: 10.21037/atm.2018.03.08
16. Feig DS, Donovan LE, Corcoy R, et al. Continuous glucose monitoring in pregnant women with type 1 diabetes (CONCEPTT): a multicentre international randomised controlled trial. Lancet 2017;390:2347-59.

17. Pickup J, Mattock M, Kerry S. Glycaemic control with continuous subcutaneous insulin infusion compared with intensive insulin injections in patients with type 1 diabetes: meta-analysis of randomised controlled trials. BMJ 2002;324:705.

18. Nielsen LR, Ekbom P, Damm P, et al. HbA1c Levels Are Significantly Lower in Early and Late Pregnancy. Diabetes Care 2004;27:1200-1.

19. Hughes RCE, Rowan J, Florkowski CM. Is There a Role for HbA1c in Pregnancy? Curr Diab Rep 2016;16:5.

20. Law GR, Gilthorpe MS, Secher AL, et al. Translating HbA1c measurements into estimated average glucose values in pregnant women with diabetes. Diabetologia 2017;60:618-24.

21. Denison FC, MacGregor H, Stirrat LI, et al. Does attendance at a specialist antenatal clinic improve clinical outcomes in women with class III obesity compared with standard care? A retrospective case-note analysis. BMJ Open 2017;7:e015218.

22. Miller KM, Beck RW, Bergenstal RM, et al. Evidence of a Strong Association Between Frequency of Self-Monitoring of Blood Glucose and Hemoglobin A1c Levels in T1D Exchange Clinic Registry Participants. Diabetes Care 2013;36:2009-14.

23. Chow N, Shearer D, Aydin Plaa J, et al. Blood glucose self-monitoring and internet diabetes management on A1C outcomes in patients with type 2 diabetes. BMJ Open Diabetes Res Care 2016;4:e000134. 